\title{
Educación virtual en la universidad en tiempos de covid-19
}

\section{Fabián Eugenio Bravo Guerrero}

Autor para la correspondencia: fabian.bravo@ucuenca.edu.ec

Orcid: $\quad$ https://orcid.org/0000-0002-0372-2071

Universidad de Cuenca, Ecuador

\section{Tatiana Gabriela Quezada Matute}

Autor para la correspondencia: tatiana.quezada@ucuenca.edu.ec

Orcid: https://orcid.org/0000-0003-2730-9342

Universidad de Cuenca, Ecuador

Fecha de recepción: 8 de octubre del 2020

Fecha de aceptación: 24 de enero del 2021

\section{Resumen}

Con la irrupción de la pandemia del Covid-19 el mundo atraviesa por cambios bruscos, la población debió aislarse y la educación tuvo que pasar de la modalidad presencial hacia la virtualidad. Esta abrupta transición y la posterior adaptación al nuevo sistema han traído muchas dificultades a los estudiantes, entender esta problemática fu el objetivo de esta investigación, porque esta nueva información que se genera sirve para mejorar los procesos virtuales de enseñanza y aprendizaje. La investigación tuvo un enfoque cuantitativo, la técnica usada fue la encuesta y como instrumento un cuestionario estructurado aplicado a 176 estudiantes universitarios de estudiantes de la carrera de formación docente en Matemáticas y Física de la Universidad de Cuenca, Ecuador. Se encontró que el alumnado tiene dificultades con la conectividad y atraviesan problemas económicos; para sus estudios atienden en promedio a 11,6 horas de clases virtuales a la semana y dedican 15,7 horas semanales a desarrollar sus tareas, consideran que las clases virtuales no son suficientes y deben complementar sus estudios con aprendizaje autónomo. Los docentes y estudiantes están todo el tiempo mejorando sus procesos de enseñanza y aprendizaje en la modalidad virtual, con esta investigación se prendió conocer cómo se dan las cosas en este tiempo atípico de pandemia.

Palabras clave: COVID-19; estudiantes universitarios; modalidad virtual

\section{Abstract}

With the emergence of the Covid-19 pandemic, the world is going through sudden changes, the population had to isolate itself and education had to move from face-to-

Esta obra se comparte bajo la licencia Creative Common Atribución-No Comercial 4.0 
face to virtuality. This abrupt transition and the subsequent adaptation to the new system have brought many difficulties to the students, understanding this problem is the objective of this research, because this new information that is generated serves to improve the virtual teaching and learning processes. Research has a quantitative approach, the technique used was the survey and as an instrument a structured questionnaire applied to 176 university students from a teacher training career. It was found that students have difficulties with connectivity and are experiencing financial problems; for their studies, they attend an average of 11.6 hours of virtual classes a week and dedicate 15.7 hours a week to developing their homework. They consider that virtual classes are not enough and should complement their studies with autonomous learning. Teachers and students are constantly improving their teaching and learning processes in the virtual mode, with this research we want to know how things happen in this atypical time of a pandemic.

Keywords: COVID-19; university students; virtual mode

\section{Introducción}

La pandemia del Covid-19 trajo cambios imprevistos al mundo y de forma particular a la educación, el propósito de esta investigación es conocer las dificultades atraviesan los estudiantes universitarios y cómo se han adaptado a estudiar en la modalidad virtual. Es trascendental conocer cómo ha transcurrido esta etapa, porque la modalidad de educación virtual continúa y deben ser mejorados estos procesos para el futuro. Dado que el virus Sars Cov-2 infectó a los humanos desde fines del 2019, hay poco conocimiento del tema, la información se va generando a partir de investigaciones recientes.

A partir de marzo del 2020 en el Ecuador se decretó la medida de confinamiento de la población para intentar controlar la diseminación de la enfermedad Covid-19, los estudiantes universitarios habían iniciado su semestre apenas una semana antes en la modalidad presencial, y súbitamente debieron pasar a la modalidad de estudios virtual. Ante esta nueva realidad, se plantean tres preguntas que orientan esta investigación, ¿qué dificultades atravesaron los estudiantes en sus hogares con la pérdida de ingresos y complicaciones con sus estudios?, ¿qué problemas tuvieron con los dispositivos electrónicos y la conectividad?, y posteriormente, cuando las cosas fueron tomando su curso, ¿cómo los profesores fueron adaptándose a la nueva modalidad?.

Se quiere conocer las dificultades que atraviesan los estudiantes en sus hogares, los problemas que tienen para acceder a las clases en la modalidad virtual, y cómo los docentes están desarrollando sus clases virtuales. La temática investigada es importante porque este cambio forzado de modalidad ha abierto un nuevo campo, el de la 
DOI: $10.33970 /$ eetes.v5.n1.2021.238 educación virtual, a partir de esta información generada, se pueden tomar decisiones que hagan de esta una modalidad exitosa.

\section{Marco Teórico}

Las nuevas tecnologías han tenido un vertiginoso desarrollo en los últimos años y han pasado a ser un recurso indispensable en la vida de las personas, como una herramienta que facilita la comunicación y el acceso al conocimiento. Un 60,7\% de los ecuatorianos, en promedio, tiene acceso a internet (CAF, 2020a), pero debido a las desigualdades sociales no todos logran acceder a las tecnologías y solo un $40 \%$ de la población más pobre, perteneciente al primer quintil, tiene una conexión a internet (CEPAL, 2020). Sin embargo, a pesar de que la mayoría tiene acceso a internet, su uso no se destina prioritariamente a la educación, en Ecuador se descargan solo 9,09 aplicaciones (apps) educativas al año, cuando en Latinoamérica se descargan 51,02 apps anuales, y en países desarrollados la cifra llega a 76,07 apps anuales descargadas (CAF, 2020a).

El mundo vive una crisis sanitaria que ha afectado gravemente a las personas y a la economía de varios países (CEPAL, 2020), causada por la enfermedad del coronavirus (Covid-19) que tuvo su inicio en China a finales del 2019, el 11 de marzo del 2020 la Organización Mundial de la Salud (OMS) declaró al Covid-19 como una pandemia (Hernández, 2020), se decretaron medidas de distanciamiento social y confinamiento, que llevaron a la población a aislarse en sus hogares para evitar el contagio (Cóndor-Herrera, 2020). Esto ocasionará, según el Fondo Monetario Internacional una fuerte recesión a nivel mundial en el año 2020 (CAF, 2020b), que traerá dificultades a las economías de los países, especialmente para Latinoamérica, porque la producción se desacelera y esto lleva a la disminución del trabajo y los ingresos de las personas, impactando especialmente a los sectores vulnerables (CEPAL, 2020), en el caso de Ecuador, con una caída del Producto Interno Bruto (PIB) estimada entre un 7,3\% hasta un 9,6\% en el escenario más desfavorable (Tapia, 2020).

Dado que el mayor impacto tienen las actividades que dependen del contacto interpersonal, se forzó una rápida virtualización de estas actividades, entre ellas la educación (CEPAL, 2020), para el 20 de marzo se habían suspendido las clases en la mayoría de los países de Latinoamérica, forzando a la educación a encontrar alternativas para continuar con sus labores a la distancia, fue entonces que las TIC se convirtieron en un recurso indispensable para continuar con esa modalidad de educación. Pero, las universidades no tenían experiencia en la modalidad virtual (Torrecillas, 2020), ni tuvieron tiempo de adecuar o rediseñar las asignaturas, ni asegurarse que docentes y estudiantes contaran con los medios tecnológicos para el cambio forzado (GarcíaPeñalvo, Corell, Abella-García, \& Grande, 2020), por lo que, tuvieron que tomar decisiones en la marcha, definiendo horarios, metodologías, técnicas evaluación (Toledo-Ibarra, et al., 2020), entre otros.

Esta obra se comparte bajo la licencia Creative Common Atribución-No Comercial 4.0 
DOI: $10.33970 /$ eetes.v5.n1.2021.238

En el Ecuador se decretó la emergencia sanitaria por el Covid-19 el 11 de marzo del 2020, suspendiendo sus actividades todas las universidades del país, con sus 750.000 estudiantes, y 100.000 docentes y administrativos (SENESCYT, 2020), para el 6 de mayo del 2020 se expidió la normativa transitoria por el estado de excepción por la emergencia sanitaria Covid-19, donde se formaliza el cambio de modalidad y se dictaron una serie de reglas y procedimientos de la nueva modalidad virtual. Allí se especifica que dadas las dificultades de acceso "la asistencia a clases no incidirá en la aprobación o reprobación del curso" (CES, 2020, Art. 11), también que "la evaluación de aprendizajes será continua, planificada y transparente" (CES, 2020, Art. 11a). Ante las dificultades que puedan suscitarse en las carreras que tienen que desarrollar prácticas pre profesionales (Velazque, Valenzuela, \& Murillo, 2020), solo pueden desarrollar sus componentes teóricos (Pavón, 2020), pero el Art. 8 norma que estas asignaturas se pueden modificar, diferir o incluso suspenderlas (CES, 2020). El gobierno anunció recortes en los presupuestos a todos los sectores, incluido la educación superior, por eso las universidades deben optimizar los escasos recursos para lograr sus objetivos educativos (Toledo-Ibarra, et al., 2020).

Las brechas sociales se hacen evidentes en esta difícil situación (Bara, 2020), los sectores menos favorecidos de la población tienen dificultades de acceso a la educación virtual (Toledo, 2020), pues, no todos tienen acceso a dispositivos electrónicos y tienen una deficiente conexión a internet (García-Peñalvo, Corell, Abella-García, \& Grande, 2020), por eso se sugiere a los docentes ser flexibles para no profundizar esas condiciones de desventaja (Toledo-Ibarra, et al., 2020), pues, la inclusión educativa tiene que reafirmarse ahora más que nunca (Zapatero, 2020). En estas circunstancias los docentes deben desarrollar otro tipo de competencias, las socioemocionales para apoyar al estudiante en las dificultades (Hernández, 2020), promoviendo estados de ánimo que ayuden a manejar la angustia, desesperación y frustración, y que promuevan la confianza, ilusión y fortaleza, los docentes además, deben garantizar la atención a la diversidad sin barreras (Otero, Calvo, \& Llamedo, 2020). Desde la enseñanza virtual, el docente debe favorecer los rasgos humanísticos, entendidos como la capacidad del maestro para motivar al estudiante y favorecer su interés y curiosidad por el descubrimiento de nuevos saberes (Torres, 2020).

Los docentes, que en la modalidad presencial han desarrollado sus clases de forma tradicional (Bravo, 2019a), han tenido dificultades para adaptarse a las clases virtuales (Cáceres, 2020), pues no todos tienen un buen dominio de las competencias tecnológicas que la situación amerita (Rogero-García, 2020), por ese motivo, han tenido que capacitarse (Bara, 2020; Cóndor-Herrera, 2020), porque muy pocos han tenido preparación previa para los entornos virtuales, y estos lo habían hecho de forma autodidacta (Otero, Calvo, \& Llamedo, 2020), hay que aprovechar la experiencia de esos docentes, y profundizar en métodos y procesos que permitan integrar las tecnologías al aprendizaje (Zapatero, 2020).

Esta obra se comparte bajo la licencia Creative Common Atribución-No Comercial 4.0 
Las instituciones han debido preparar a docentes y estudiantes para que puedan manejarse en los entornos virtuales con solvencia (Velazque, Valenzuela, \& Murillo, 2020), y han tenido un fuerte trabajo para incorporar nuevas estrategias para promover los aprendizajes en la nueva modalidad (Hernández, 2020), también han tenido que atender situaciones afectivas que atraviesan los estudiantes (Villafuerte, Bello, Pantaleón, \& Bermello, 2020), además han debido atender sus propias dificultades por el confinamiento, afectivas, sociales y económicas (Sánchez, et al., 2020).

La creatividad y adaptación han sido rápidas, desplegándose una serie de recursos y métodos adaptados a las clases virtuales (Morata, 2020), las clases deben resultar motivadoras para el estudiante (Cáceres, 2020), se le debe incentivar para que tenga interés en buscar información, investigar y así reforzar sus aprendizajes, se le estimula a tener una mayor autonomía, a organizar sus tiempos y procesos, es decir a ser estratégicos para aprender, también debe favorecer la retroalimentación para reforzar los aprendizajes (Torres, 2020). La clase debe evitar caer en la mera transferencia de información y de ofrecer una educación despersonzalizada (Villafuerte, Bello, Pantaleón, \& Bermello, 2020), pues, la enseñanza virtual minimiza el trato entre personas y el componente social (Torres, 2020), desarrollándose en soledad, sin diálogo, sin un sentido crítico (Almazán, 2020). Esa falta de presencialidad está siendo compensada con un aluvión de tareas y trabajos (Pavón, 2020), que añade una fuerte carga a los propios problemas derivados del coronavirus (Sánchez, et al., 2020).

Sobre la evaluación, se indica que valorar con nota la presencia virtual perjudica a los estudiantes que tienen dificultades de acceso (Cáceres, 2020), porque la digitalización no se ha masificado (Almazán, 2020). Se debe asegurar el uso de diferentes técnicas de evaluación, y estas deben valorar la adquisición de resultados de aprendizaje, sin embargo, ante la falta de dominio en el manejo de recursos digitales, la evaluación en la virtualidad trae incertidumbre tanto a los docentes como a los estudiantes (García-Peñalvo, Corell, Abella-García, \& Grande, 2020).

La modalidad virtual, es de gusto de los jóvenes, quienes tienen un mejor dominio de las tecnologías, sin embargo, las usan para socializar, para el ocio, y en menor medida usan internet y aplicaciones para la educación. En la práctica hay muchos estudiantes que tienen dificultades de acceso a equipos (Cóndor-Herrera, 2020), y de conexión a internet, por pertenecer a bajos niveles socio económicos (Velazque, Valenzuela, \& Murillo, 2020), tienen dificultades con la conectividad por vivir en zonas con una baja velocidad de conexión o incluso sin señal de internet (CAF, 2020b), a esto se suman las dificultades emocionales por el miedo al contagio de la enfermedad, al duelo, o a la ansiedad por los efectos de la crisis económica (Rogero-García, 2020).

Ha sido un gran esfuerzo de universidades, docentes y estudiantes para que el cambio a la modalidad virtual pueda salir adelante (Torrecillas, 2020), por su parte, los docentes deben continuar adaptando sus metodologías y los recursos para que todos los Esta obra se comparte bajo la licencia Creative Common Atribución-No Comercial 4.0 
DOI: $10.33970 /$ eetes.v5.n1.2021.238

alumnos puedan lograr sus aprendizajes (Rogero-García, 2020). Los estudiantes viven una situación complicada, y deben adaptarse rápidamente a la educación virtual y sobrellevar las dificultades que se dan en su hogar por la pandemia. Es un reto para el sistema educativo ante la situación emergente, que se logren aprendizajes significativos y también cuidar del estudiante para evitar que repruebe o deserte en este contexto (Villafuerte, Bello, Pantaleón, \& Bermello, 2020). Hay una ausencia de información sobre los efectos de la pandemia en los actores de la educación, instituciones, docentes y estudiantes (Sánchez, et al., 2020), por lo que esta investigación es pertinente.

\section{Metodología}

La investigación realizada tiene un enfoque cuantitativo, es de tipo exploratorio y descriptivo. Tiene el objetivo de investigar el impacto que ha tenido en los estudiantes universitarios el cambio de modalidad de estudios, desde las clases presenciales hacia la modalidad de estudios virtuales. El estudio es transversal, para esto se investigó a todos los estudiantes de la carrera de formación docente en Matemáticas y Física de la Universidad de Cuenca, Ecuador, matriculados en el período entre marzo y agosto del 2020, en total son ocho paralelos con 176 estudiantes.

La técnica cuantitativa utilizada fue una encuesta, para esto se elaboró un cuestionario estructurado de tipo cerrado, que averiguó datos generales, información del hogar, conectividad, clases virtuales y aprendizaje, de los cuales se obtuvieron diferentes datos estadísticos. El cuestionario fue pilotado a una muestra y sirvió para depurar el instrumento, luego fue aplicado a los estudiantes de forma virtual mediante formularios de Google la última semana de julio del 2020, contestaron el cuestionario de forma libre y voluntaria, y se les garantizó confidencialidad y anonimato a la información proporcionada; los datos fueron tabulados y analizados para elaborar un reporte que contiene tablas, gráficos y la respectiva reflexión de los hallazgos de la investigación.

\section{Resultados}

Respecto a la información general de los investigados, en la tabla 1 se observa que los estudiantes de segundo y cuarto semestre son la mayoría sumando un 63,7\% del total; también, que el $69,9 \%$ de ellos pertenece a la sección matutina. Hay un balance entre hombres $(50,6 \%)$ y mujeres $(49,4 \%)$. Con respecto a la edad, el $88,1 \%$ de los estudiantes tiene entre 17 y 24 años; finalmente, existen estudiantes que pertenecen a 6 diferentes provincias de la región sur del Ecuador, sin embargo, el 92\%, están radicados en la provincia del Azuay. Casi todos (87,5\%) se encontraban en sus hogares durante la cuarentena.

Tabla 1: Información general

\begin{tabular}{l}
\hline PREGUNTA \\
\hline CATEStre \\
Esta obra se comparte bajo la licencia Creative Common Atribución-No Comercial 4.0 \\
International (CC BY-NC 4.0) Revista Trimestral del Instituto Superior Universitario Espíritu Santo
\end{tabular}




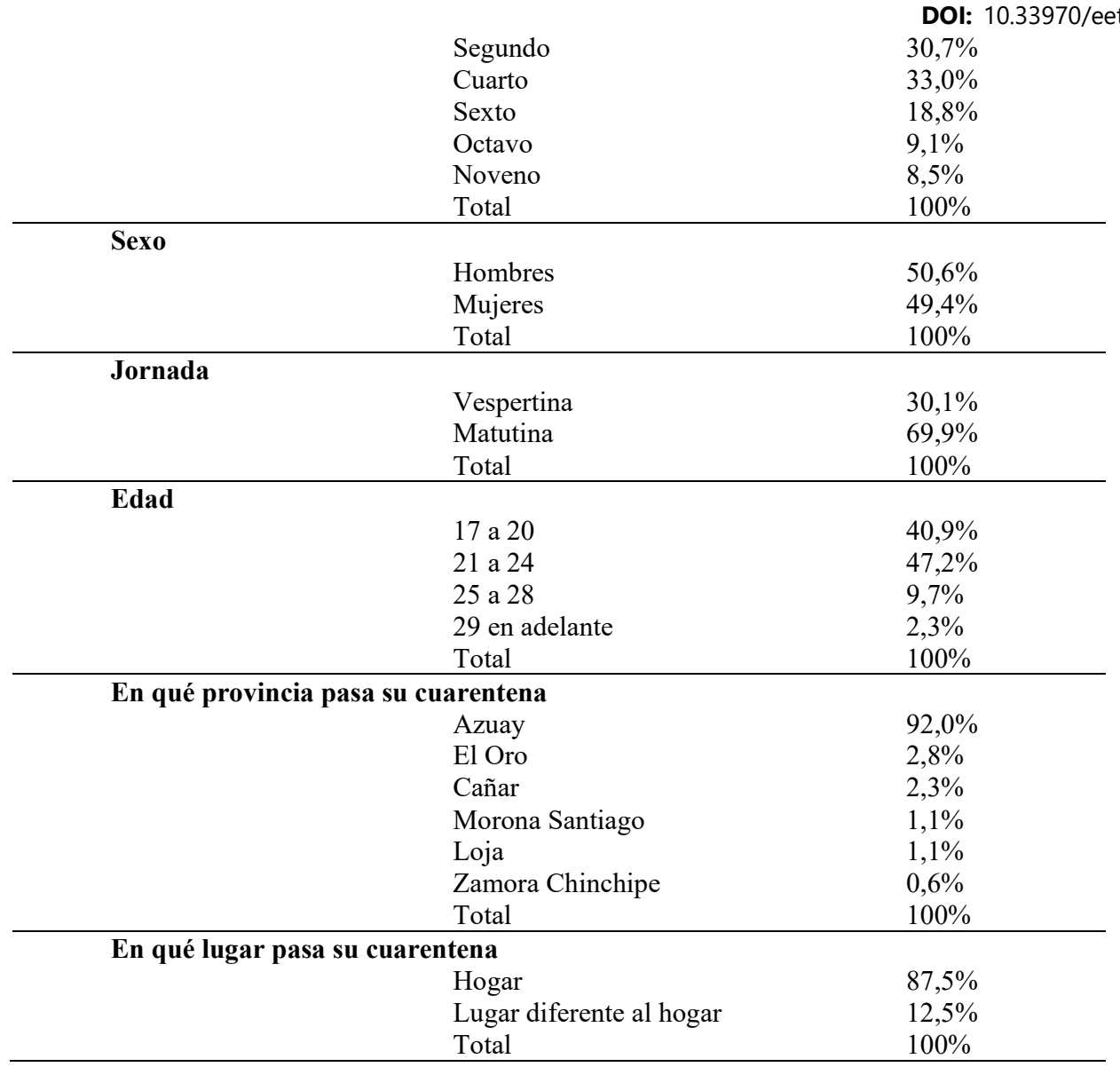

Fuente: La encuesta

Al consultarles si alguna persona cercana a ellos se ha enfermado de Covid-19 en el tiempo de confinamiento, el 36\% indica que sí; y, ante la pregunta si alguna persona cercana a ellos ha fallecido con Covid-19, indican afirmativamente el 14\% de los estudiantes.

Se les preguntó cómo está la economía en el hogar, y sólo un 7\%, asegura que las finanzas son buenas; sin embargo, el 48\% se encuentra en una situación regular y el $45 \%$ los estudiantes manifiestan que su situación económica es difícil y muy difícil, lo vemos en la figura 1. También, ante las dificultades que han debido pasar, el $40 \%$ de ellos indican que han debido conseguir un trabajo en este período. 


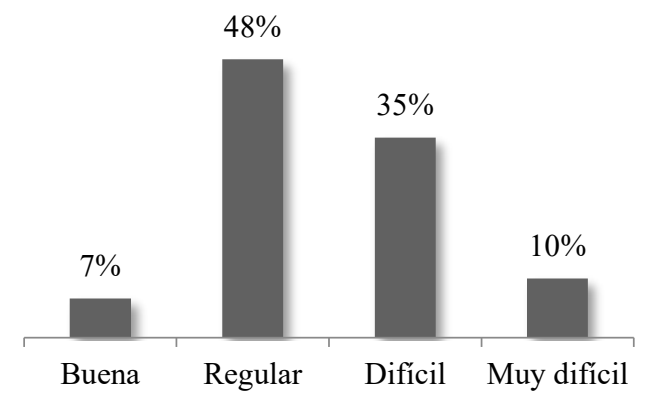

Figura 1. Cómo está la situación económica en el hogar

Fuente: La encuesta

Otra sección de la encuesta consultó sobre los dispositivos electrónicos y la conectividad, se preguntó si comparte el dispositivo con el que accede a las clases virtuales, solo el $24 \%$ tiene un dispositivo que no lo comparte, el $57 \%$ de los estudiantes manifestaron que a veces deben compartir sus dispositivos, mientras un 19\% indica que siempre comparte los dispositivos. Al preguntarles por los dispositivos más usados, tenemos el teléfono inteligente (43\%), el computador portátil (43\%), el computador de escritorio (11\%) y la tableta (4\%).

Se averiguó sobre la conectividad, y solo un $14 \%$ de la población que manifiesta que la conexión a internet es muy rápida o rápida, el 47\% tiene una conexión normal, y el 39\% asegura que la conexión que posee es lenta o muy lenta, lo apreciamos en la figura 2.

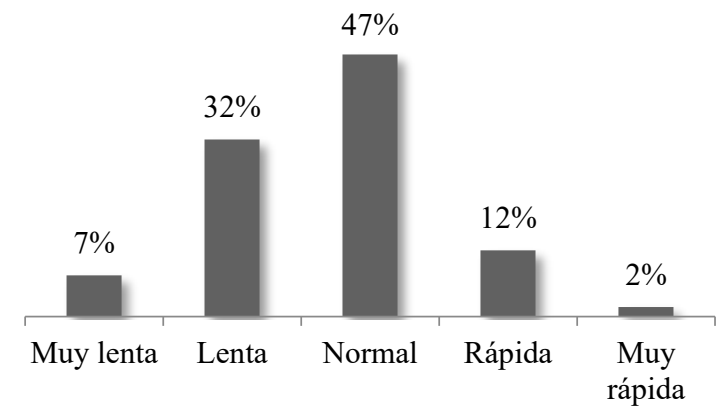

Figura 2: ¿Cómo es la conexión a internet?

Fuente: La encuesta.

El 84\% de los encuestados mencionaron que posee internet inalámbrico en su hogar, un porcentaje alto; sin embargo, el 10\% debe ir a la casa de un familiar para conectarse, el $3 \%$ de ellos se conecta por medio de su celular a través de gigas, el $2 \%$

Esta obra se comparte bajo la licencia Creative Common Atribución-No Comercial 4.0 
acude a la casa de un vecino para poder asistir a las clases virtuales, y el $1 \%$ se traslada a un café net para conectarse.

Los estudiantes indicaron que destinan a sus clases virtuales en promedio 11,6 horas semanales, con una desviación estándar de 6,9 horas semanales; también, para realizar tareas pasan en promedio 15,7 horas semanales, con una desviación estándar de 12,8 horas/semanales.

El 73\% consideró que el contacto que tiene con sus docentes no es suficiente para aprender los temas. Al preguntarles cuánto han cambiado las metodologías que usan los docentes para desarrollar sus clases virtuales, un $11 \%$ indica que poco o nada han cambiado, y un $48 \%$ dice que las metodologías cambiaron mucho o totalmente, lo apreciamos en la figura 3 .

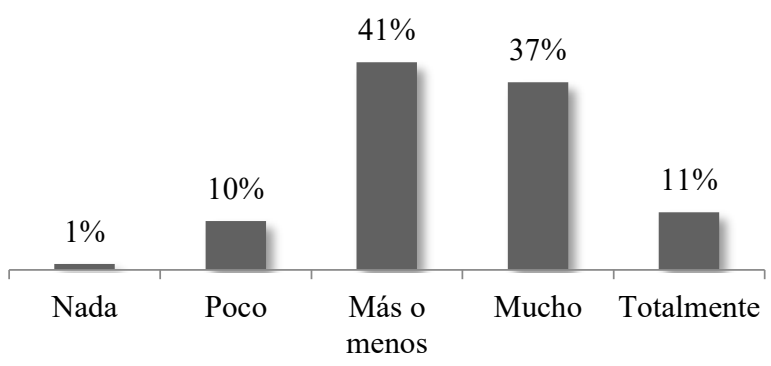

Figura 3: Cuánto han cambiado las metodologías de los docentes.

Fuente: La encuesta.

Sobre los recursos que los docentes usaron en las clases virtuales hay variedad, siendo los más frecuentes las diapositivas ( $90 \%$ lo usan), las clases en línea ( $89 \%$ lo usan), las clases grabadas ( $72 \%$ lo usan), todos estos propios de la modalidad virtual, y otros recursos, cuyo detalle lo apreciamos en la figura 4.

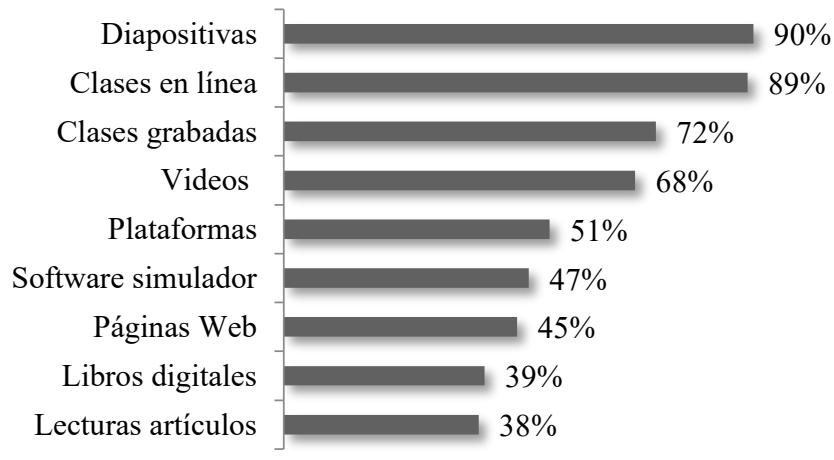

Figura 4: Frecuencia de uso de recursos en las clases virtuales

Esta obra se comparte bajo la licencia Creative Common Atribución-No Comercial 4.0

International (CC BY-NC 4.0) Revista Trimestral del Instituto Superior Universitario Espíritu Santo 
Fuente: La encuesta.

Al preguntarles si el aprendizaje autónomo ayudó a complementar las clases virtuales, el $99 \%$ está de acuerdo, y solo el 1\% de los encuestados mencionó que no les ayuda. Finalmente se les consultó cuánto logran aprender de las clases virtuales, y un $21 \%$ indica que aprendió poco o nada, el $53 \%$ más o menos, y un $26 \%$ indicó que mucho o totalmente, como lo vemos en la figura 5.

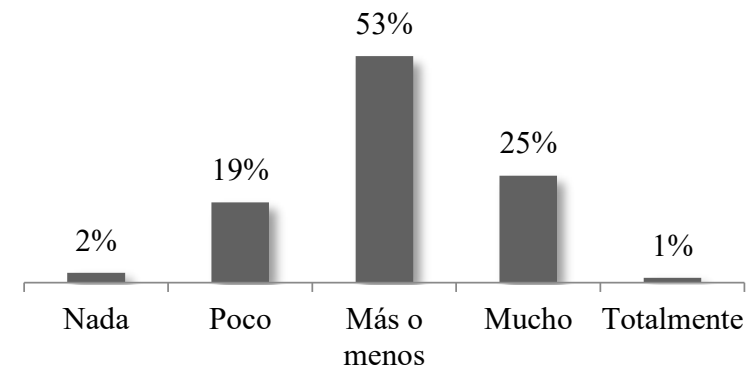

Figura 5: Cuánto logró aprender de las clases virtuales

Fuente: La encuesta.

\section{Discusión}

En esta investigación, se halló que muchos estudiantes tienen dificultades al acceder a clases virtuales de forma sincrónica, no todos tienen un dispositivo propio, un $43 \%$ lo hace desde un teléfono, y muchas veces lo tienen que compartir con alguien más (76\%); además, no tienen señal de internet y deben movilizarse para conseguirla, en otros casos la calidad de la señal es deficiente (39\%). Estos hallazgos tienen similitud con las dificultades de acceso a dispositivos tecnológicos que expresa Cóndor-Herrera (2020), y de conectividad que lo mencionan Velazque, Valenzuela y Murillo (2020).

Hernández (2020) recomienda que en la virtualidad los docentes deban tener destreza en el manejo de las TIC y también desarrollar competencias socioemocionales, para ayudar a los estudiantes a sortear las dificultades que han debido que pasar con la pandemia; según Rogero-García (2020) los estudiantes han atravesado por problemas debido a la enfermedad, duelo y ansiedad. En el caso de esta investigación, los estudiantes están pasando por dificultades, como tener personas cercanas enfermas $(36 \%)$ o fallecidas (14\%) por Covid-19, otros han tenido que buscar un trabajo (40\%) para afrontar las carencias económicas.

Aunque los docentes tradicionales han tenido limitaciones en el manejo de recursos tecnológicos y siempre han usado la pizarra (Bravo, 2019b), el cambio de modalidad los ha obligado a innovar y usar una serie de recursos tecnológicos que les ha Esta obra se comparte bajo la licencia Creative Common Atribución-No Comercial 4.0 International (CC BY-NC 4.0) Revista Trimestral del Instituto Superior Universitario Espíritu Santo 
DOI: $10.33970 /$ eetes.v5.n1.2021.238

permitido adaptarse a las clases virtuales (Morata, 2020), como en el caso de esta investigación, que los profesores han elaborado diapositivas $(90 \%)$, desarrollado clases virtuales $(89 \%)$, grabado sus clases $(72 \%)$, usado plataformas $51 \%)$, software simulador (47\%), consultado documentos digitales (39\%), entre otros nuevos recursos.

\section{Conclusiones}

La pandemia ha alterado la vida de las personas, muchos estudiantes tienen personas cercanas con la enfermedad, algunos incluso tienen parientes fallecidos, el confinamiento ha causado la pérdida de ingresos en los hogares ocasionando situaciones que ellos califican como difíciles, incluso algunos estudiantes han tenido que buscar un trabajo para ayudar a la economía. Por eso es importante que el docente trabaje en la motivación de sus estudiantes.

A pesar de que una buena parte de los estudiantes posee internet en sus hogares, muchos indican que la conectividad no es buena, otros deben recibir sus clases desde un teléfono inteligente, y algunos deben compartir sus dispositivos con más personas del hogar. Esta realidad sugiere que los docentes podrían grabar las clases para que sean revisadas por los estudiantes en cualquier momento.

El cambio de modalidad ha hecho que la metodología del docente también varíe, ahora ha tenido que usar recursos tecnológicos para desarrollar sus clases virtuales, sin embargo los estudiantes indican que necesitan más horas en contacto con el docente, casi todos están de acuerdo que el trabajo autónomo les ayuda a comprender los temas, aunque al final indican que han logrado un nivel intermedio de aprendizaje de los temas. Atender a estas dificultades, sin duda mejorará los procesos de educación virtual.

\section{Referencias bibliográficas}

Almazán, A. (2020). Covid-19: ¿Punto Sin Retorno de la Digitalización de la Educación?. Revista Internacional de Educación para la Justicia Social, 9(3), $1-4$.

Bara, F. (2020). COVID-19: la universidad debe renovarse en un entorno virtual sin perder su esencia. The Conversation, 1, 1-3.

Bravo, F. (2019a). Dificultades que enfrentan los nuevos estudiantes universitarios en matemática. INNOVA Research Journal, 5(1). 1-13.

Bravo, F. (2019b). Las nuevas clases de geometría. RECUS Revista Electrónica Cooperación Universidad Sociedad, 4(3), 14-21.

Esta obra se comparte bajo la licencia Creative Common Atribución-No Comercial 4.0 
Cáceres, K. (2020). Educación virtual: creando espacios afectivos de convivencia y aprendizaje en tiempos de covid-19. CienciAmérica, 9(2).

CEPAL. (2020). América Latina y el Caribe ante la pandemia del COVID19. Efectos económicos y sociales. Comisión Económica Para América Latina.

CES. (2020). Normativa transitoria para el desarrollo de actividades académicas en las Instituciones de Educación Superior, debido al estado de excepción decretado por la emergencia sanitaria ocasionada por la pandemia de COVID-19. Quito: Consejo de Educación Superior.

Corporación Andina de Fomento. (2020a). El estado de la digitalización de América Latina frente a la pandemia del COVID-19. CAF, Banco de Desarrollo de América Latina.

Corporación Andina de Fomento. (2020b). Las oportunidades de la digitalización en América Latina frente al Covid-19. CAF, Banco de Desarrollo de América Latina.

Cóndor-Herrera, O. (2020). Educar en tiempos de Covid-19. CienciAmérica, $9(2)$.

García-Peñalvo, F., Corell, A., Abella-García, V., \& Grande, M. (2020). Online Assessment in Higher Education in the Time of COVID-19. Education in the Knowledge Society, 12, 1-26.

Hernández, A. R. (2020). Covid-19: El efecto en la gestión educativa. Relais, 3(1), 37-41.

Morata, J. (2020). Uso de TIC en orientación educativa en tiempos de COVID-19. AOSMA, 1, 88-91.

Otero, L., Calvo, M. I., \& Llamedo, R. (2020). Herramientas digitales para la comunicación, la tele-docencia y la tele-orientación educativa en tiempos de COVID-19. AOSMA, 1, 92-103.

Pavón, A. (2020). La impotencia de la pedagogía posmoderna frente a la crisis del coronavirus. El Catoblepas, 191, 15-26.

Rogero-García, J. (2020). La ficción de educar a distancia. Revista de Sociología de la Educación-RASE, 13(2), 174-182.

Sánchez, M., Martínez, A., Torres, R., de Agüero, M., Hernández, A., Benavides, M., Rendón, V., \& Jaimes, C. (2020). Retos educativos durante la pandemia de COVID-19: una encuesta a profesores de la UNAM. Revista Digital Universitaria, 21(3), 1-24.

Esta obra se comparte bajo la licencia Creative Common Atribución-No Comercial 4.0 
SENESCYT. (2020). Universidades continúan sus actividades mediante aulas virtuales. (Boletín de prensa \#22). Recuperado de https://www.educacionsuperior.gob.ec/universidades-continuan-sus-actividadesmediante-aulas-virtuales/

Tapia, E. (2020). La economía ecuatoriana puede caer hasta 9,6\% en este 2020. El Comercio. Recuperado de https://www.elcomercio.com/actualidad/economia-ecuador-banco-centraldolarizacion.html

Toledo-Ibarra et al., (2020). The COVID-19 pandemic from the multidisciplinary vision of 28 University Professors of Nayarit, Mexico. Revista Bio Ciencias, 7, 1-33.

Torrecillas, C. (2020). El reto de la docencia online para las universidades públicas españolas ante la pandemia del Covid-19. ICEI Papers COVID-19, 16, 14.

Torres, J. (2020). Propuestas para el mejoramiento de la educación universitaria virtual después del brote del COVID-19. UVserva, 1, 4-7.

Velazque, L., Valenzuela, C., \& Murillo, F. (2020). Pandemia COVID-19: repercusiones en la educación universitaria. Odontología Sanmarquina, 23(2), 203-206.

Villafuerte, J., Bello, J., Pantaleón, Y., \& Bermello, J. (2020). Rol de los docentes ante la crisis del Covid-19, una mirada desde el enfoque humano. REFCalE, 8(1), 134-150.

Zapatero, J. (2020). La orientación educativa en tiempos de COVID-19: con ganas, en equipo y con humildad. AOSMA, 1, 28-32. 